\title{
The effect of early tranexamic acid administration on hemoglobin levels after unstable pelvic fracture: An experimental study in rats
}

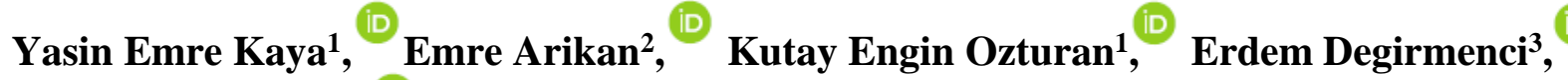 \\ Tacettin Ayanoglu, ${ }^{1}$ Ozgur Mehmet Yis ${ }^{4}$ \\ ${ }^{I}$ Department of Orthopedics and Traumatology, Bolu Abant Izzet Baysal University, Faculty of Medicine, Bolu, Turkey \\ ${ }^{2}$ Department of Orthopedics and Traumatology, Canakkale Mehmet Akif Ersoy Public Hospital, Çanakkale, Turkey \\ ${ }^{3}$ Department of Orthopedics and Traumatology, Private Duzce Cagsu Hospital, Duzce, Turkey \\ ${ }^{4}$ Department of Medical Biochemistry, Bolu Abant Izzet Baysal University, Faculty of Medicine, Bolu, Turkey
}

\section{ABSTRACT}

Aim: To investigate the effect of early systemic tranexamic acid (TRA) administration on hemoglobin (Hb) levels in rats with pelvic fracture.

Method: In our study, 30 Wistar Albino rats were randomly divided into 3 groups in equal numbers and their hemoglobin levels were measured by taking blood samples from each. No trauma was applied to the first group and it was taken as the main control group of the study. Closed bilateral unstable pelvic fractures were created in all rats in groups 2 and 3. Fracture creation time is considered as minute 0.10 minutes after the fracture was formed, TRA was given to the 1st group, TRA to the 2nd group and saline solution to the 3rd group systemically. Hemoglobin levels were measured by taking blood samples from all rats at 30th minute and 24th hour. The initial $\mathrm{Hb}$ values obtained were normalized to 100 and the percentages of 30th minute and 24th hour values were calculated. The initial, 30th minute and 24th hour values of all groups were compared statistically with each other. The 30th minute and 24th hour values were compared statistically between the groups.

Results: No death was observed within 24 hours in all three groups. When the first Hb values of each group are normalized to 100 , the mean $\mathrm{Hb}$ percentages were calculated in the first group as 99.54 and 99.84 at 30 minutes and 24 hour, respectively; 92.95 and 87.73 in the second group; and 87.95 and 73.16 in the third group. When these values obtained were compared statistically within the groups (initial, 30th minute, 24th hour $\mathrm{Hb}$ percentages), there was no significant difference between the initial, 30th minute and 24th hour values in group 1. However, a statistically significant difference was found between the initial, 30th minute and 24th hour values in group 2 and $3(p<0.01)$. In the comparison between the groups, a statistically significant difference was found between group 1-2, group 1-3 and group 2-3 between both 30th minute and 24th hour values $(p<0.01)$.

Conclusion: In rats with bilateral unstable pelvic fractures due to blunt pelvis trauma, early administration of TRA after trauma significantly reduced the first 24-hour decrease in Hb value. Our study supports the early and prehospital use of TRA in traumas that are predicted to progress with acute bleeding, such as unstable pelvic fractures.

Key words: Blunt pelvic fracture, hemorrhage, hemoglobin level, tranexamic acid, rat.

$\triangle D$ r. Yasin Emre Kaya, Department of Orthopedics and Traumatology, Bolu Abant Izzet Baysal University, Faculty of Medicine, Bolu, Turkey
E-mail: yemrek@hotmail.com

Received: 2021-06-07 / Revisions: 2021-08-11

Accepted: 2021-08-27 / Published online: 2021-10-01 


\section{Introduction}

One in ten deaths worldwide are caused by traumatic injuries, and uncontrolled bleeding is responsible for almost half of these traumatic deaths [1]. Pelvic ring fractures occur as a result of high-energy trauma and constitute 1.5 to 3 percent of all fractures [2]. As is known, pelvic ring fractures are fractures with high mortality and morbidity. Although accompanying additional injuries such as head trauma, chest trauma, and abdominal injuries are among the main factors that highlight mortality in patients with pelvic fractures, it has been stated that the main reason that increases mortality in isolated pelvic fractures is massive hemorrhage [3-7]. Considering all types of pelvic fractures, mortality is seen at a rate of $5-10 \%$. It has been reported that this rate reaches up to $60 \%$ when it is accompanied by hemodynamic instability [8,9]. Despite the advancement and development of innovative multidisciplinary approaches in trauma management, posttraumatic bleeding emerges as the main cause of death in the first 24 hours after trauma [10].

Tranexamic acid (TRA) is the synthetic analogue of lysine amino acid. It shows antifibrinolytic effect by binding to plasminogen or plasmin through lysine receptors. With this effect, it prevents plasmin from binding to fibrin and preserves the matrix structure of fibrin [11]. The article on the first clinical use of TRA was published in 1968 regarding its use in the treatment of severe menstrual bleeding [12]. In the course of time, its use in dental, urological, cardiac and transplant surgeries has become widespread, and its use in arthroplasty and spinal surgeries in the orthopedic field has been accepted. Its use in patients with polytrauma and uncontrolled bleeding has been investigated, and studies on its use to reduce the amount of bleeding in patients with polytrauma in both prehospital and emergency services gained popularity. In this animal experiment we conducted, we aimed to shed light on the use of TRA in the early post-traumatic period in patients with life-threatening pelvic fractures due to severe bleeding by observing the effect of early TRA administration on hemoglobin levels in rats with pelvic fracture created by blunt pelvis trauma.

\section{Materials and methods}

This study was conducted with the ethical approval given by Animal Experiments Local Ethics Committee at Bolu Abant Izzet Baysal University (approval number 2018/19). Thirty female Wistar Albino rats, 2-4 months old and weighing 200-250 g, were used in our study. Rats were randomly divided into 3 groups with 10 rats in each group and the rats were numbered from 1 to 30 . Blood samples were taken from all rats under general anesthesia to measure the hemoglobin levels of the rats. For general anesthesia, ketamine/xylazine was administered to each rat at $90 / 10 \mathrm{mg} / \mathrm{kg}$ intramuscularly (im). 0.25-0.5 ml blood sample was taken from the right periorbital venous bed of anesthetized rats with the help of a glass capillary tube into blood collection tubes containing ethylenediaminetetraacetic acid (Mini Collect Complete K2EDTA Greiner Bioone) and sent to the biochemistry laboratory for complete blood count. After the first blood was taken, no pelvis trauma was applied to the rats in group $1(n=10)$ which were designated as the control group. In group $2(n=10)$ and group 3 $(n=10)$, a closed bilateral unstable pelvic fracture was created under general anesthesia (ketamine/xylazine, 90/10 mg/kg im). In order to create a pelvic fracture and to expose the rats to a standard trauma as possible, a weight dropping device was used, which allows the 


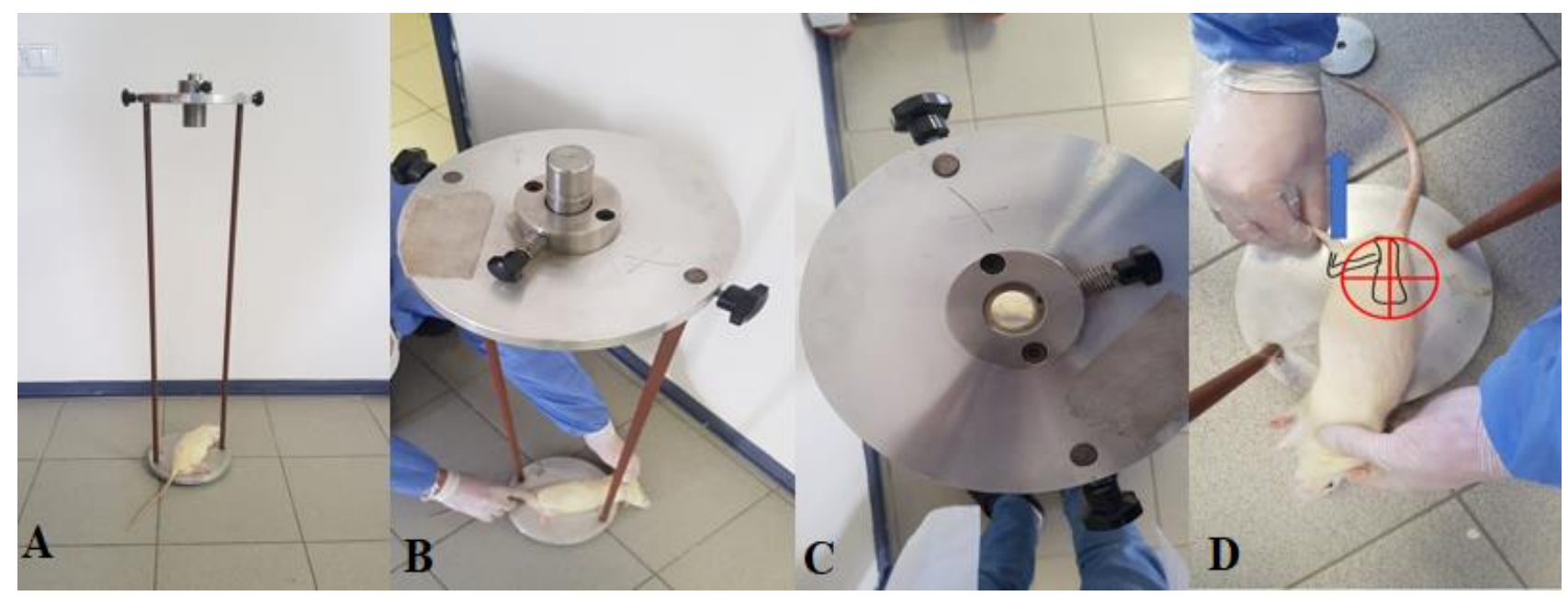

Figure 1. A) Weight dropping device that allows the metal cylindrical block weighing $100 \mathrm{~g}$ to fall upon the desired point from a height of 1 meter. The distance between the lower and upper table is 1 meter. The diameters of the upper and lower round trays are equal to each other. B) Image of the anesthetized rat was placed on the lower round metal plate in the left lateral decubitus position and the pelvis in the middle of the lower round table. C) The image of the $2 \mathrm{~cm}$ diameter cylindrical channel in the center of the upper plate through which the cylindrical metal block with a diameter of $2 \mathrm{~cm}$ can pass. D) Demonstrative view of the target. We took care to match the falling cylinder base between the most proximal point of the iliac wing and the trochanteric protrusion (red target symbol) which can be felt by palpation.

metal cylindrical block weighing $100 \mathrm{~g}$ to fall upon the desired point on rat from a height of 1 meter (Figure 1A).

The anesthetized rat was placed on the lower round metal plate in the left lateral decubitus position and the pelvis in the middle of the lower round table, as seen in Figure 1B. A metal cylinder weighing $100 \mathrm{~g}$ was placed in the channel in the middle of the upper table and the metal cylinder was released from a distance of 1 meter and dropped onto the pelvis of the rat placed in the lateral decubitus position on the lower plate (Figure 1C). It was taken care to match the falling cylinder base between the most proximal point of the iliac wing and the trochanteric protrusion which can be felt by palpation (Figure 1D).

The presence of instability in both hemipelvis was confirmed by the movement of the hemipelvis in the axial, coronal and sagittal planes with pelvic examination. In 4 rats $(2$ in group 2 and 2 in group 3 ) in which complete instability could not be felt in the pelvic examination after weight release from height method, the fracture was displaced and instability was achieved by the same researcher with slight manipulation to the hemipelvis, where instability could not be felt completely, and pathological movement and crepitation were made noticeable by physical examination. Pelvic fractures were demonstrated by anteroposterior (AP) pelvis radiographs (Figure 2A). In cases where the fracture could not be seen with AP pelvis radiographs, the iliac oblique pelvis radiographs were taken to reveal the fractures (Figure 2B, C).

This sequence of methods used to create fractures was repeated in the same way in all rats in groups 2 and 3 . In the rats in groups 2 and 3 , the fracture moment was accepted as 0 minutes. At the 10th minute, rats in groups 1 and 2 were administered intraperitoneally by diluting $30 \mathrm{mg} / \mathrm{kg}$ TRA in $1 \mathrm{cc}$ of saline solution. Rats in group 3 were given only $1 \mathrm{cc}$ 


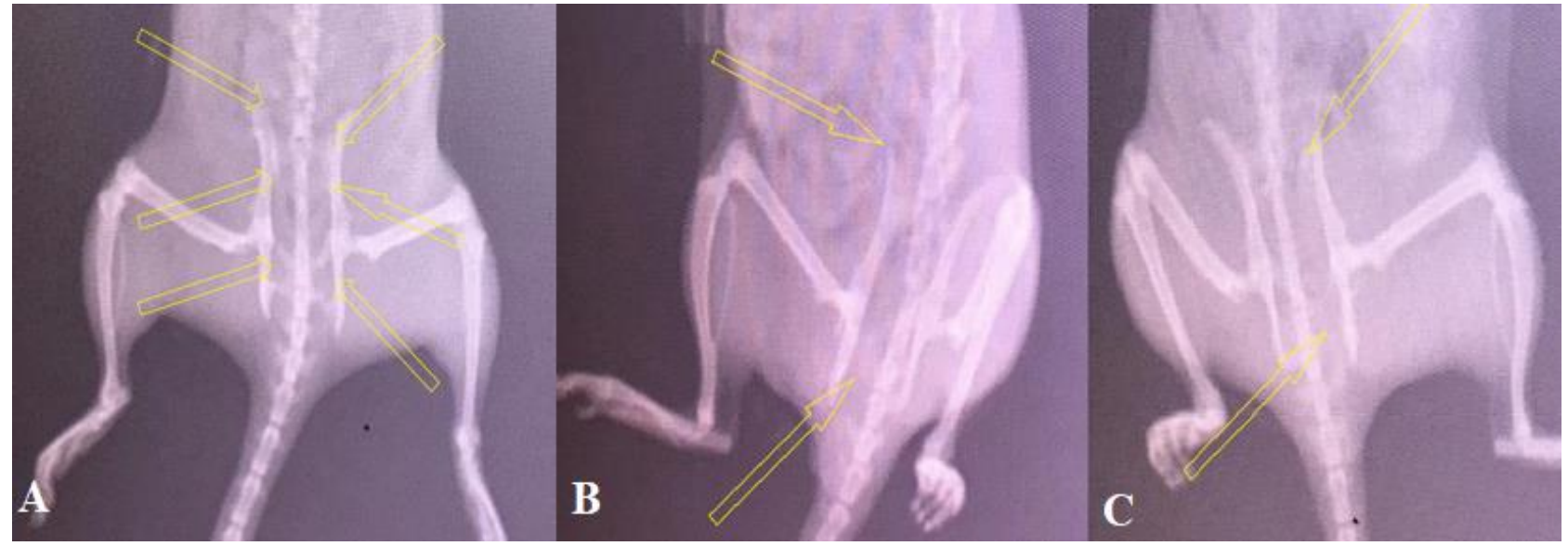

Figure 2. A) AP pelvis radiograph of a rat after trauma. B, C) Iliac oblique radiographies of a rat after trauma.

of saline solution intraperitoneally. All rats in all three groups were administered metamizole sodium $50 \mathrm{mg} / \mathrm{kg}$ intramuscularly as a pain reliever.

At the 30th minute, blood samples were collected from all rats under general anesthesia (ketamine/xylazine, 90/10 $\mathrm{mg} / \mathrm{kg}$ i.m.), this time from the left periorbital venous beds with the same method for complete blood count. The survival of all rats was monitored until the 24th hour. All rats were fed ad libitum for 24 hours. At the 24th hour, under general anesthesia (ketamine/xylazine, 90/10 $\mathrm{mg} / \mathrm{kg} \quad$ i.m.), thoracotomy was made and ventricular blood discharge was performed on all rats and the rats were sacrificed. With this method, the last blood samples were taken for complete blood count. All of the blood samples taken were studied on the Cell Dyn 3700 (Abbott, IL, USA) device on the same day. The hemoglobin values obtained were recorded.

The first hemoglobin value of each rat was normalized to 100 and the percentages of 30th minute and 24th hour values were calculated. With the one-way Anova and Tukey HSD tests, the initial, 30th minute and 24th hour values of each group were statistically compared with each other within the group itself. The 30th minute and 24th hour values were compared between the groups using the same statistical methods.

\section{Statistical analyses}

Analysis of all data groups are performed in a sequence as one way analysis of variance ANOVA and Tukey multiple comparisons to test if any significant differences between the observed group of samples in $0 \mathrm{~min}, 30 \mathrm{~min}$ and 24 hours which is A, B and C. The p-value corresponding to the F-statistic of one-way ANOVA is expected to be $<0.05(5 \%)$ for indication significant difference of means, and Q-statistic - value for Tukey test is expected to be $<0.01(1 \%)$ for indication of significant difference between the par of groups (A vs B, $\mathrm{A}$ vs $\mathrm{C}, \mathrm{B}$ vs $\mathrm{C})$. All analysis are performed using JASP (JASP Team (2020). JASP (Version 0.14.1) Computer software).

\section{Results}

No death was observed within 24 hours in all three groups. When the initial hemoglobin values of each group are normalized to 100 (initial value), the average hemoglobin percentages in the first group at 30th minute and 24th hour were respectively 99.54 and 99.84 ; 92.95 and 87.73 in the second group; in the 3rd group, it was calculated as 87.95 and 73.16 
Table 1. Mean values of $\mathrm{Hb}$ levels (initial, 30th minute and 24th hour) and ratio of 30th minute and 24th hour values to initial values.

\begin{tabular}{|c|c|c|c|c|c|c|c|}
\hline Groups & $\begin{array}{l}\text { Initial } \\
\mathrm{Hb} \text { (g/dl) }\end{array}$ & $\begin{array}{l}\text { Normalized } \\
\text { value } \\
\text { (to 100) }\end{array}$ & $\begin{array}{l}3^{\text {th }} \text { minute } \\
\mathrm{Hb}(\mathrm{g} / \mathrm{dl})\end{array}$ & $\begin{array}{l}30^{\text {th }} \text { minute } \\
\% \text { ratio }\end{array}$ & $\begin{array}{l}24^{\text {th }} \text { hour } \\
\text { Hb (g/dl) }\end{array}$ & $\begin{array}{l}24^{\text {th }} \text { hour } \\
\% \text { ratio }\end{array}$ & $P$ \\
\hline Group 1 & 15.20 & 100 & 15.13 & 99.54 & 15.17 & 99.84 & $<0.01 * *$ \\
\hline Group 2 & 14.79 & 100 & 13.75 & 92.95 & 12.98 & 87.73 & $<0.01 * *$ \\
\hline Group 3 & 15.28 & 100 & 13.44 & 87.95 & 11.19 & 73.16 & $<0.01 * *$ \\
\hline$p$ & & & & $<0.01 *$ & & $<0.01 *$ & \\
\hline
\end{tabular}

*shows the difference between group 1, 2 and 3 at 30th minute and 24th hour

**shows the difference between initial, 30th minute and 24th hour values in group 1, 2 and 3.

(Table 1). When these values were compared statistically with the one-way Anova test and Tukey HSD test within the groups (initial, 30th minute and 24th hour hemoglobin percentages), no significant difference was found between the initial, 30th minute and 24th hour values in group 1 ( $p>0.01)$. A statistically significant difference was found between the initial, 30th minute and 24th hour values in group 2 (initial> 30th minute $>24$ th hour values, $p<0.01$ ).

A statistically significant difference was found between the initial, 30th minute and 24th hour values in group 3 (initial> 30th minute> 24th hour values, $p<0.01$ ). When 30th minute values were compared between the groups, a statistically significant difference was found between group 1, group 2 and group 3 (group 1> group 2> group 3, $p<0.01$ ).

When the 24th hour values were compared between the groups, a statistically significant difference was found between group 1, group 2 and group 3 (group 1> group 2> group 3,p $<0.01)$. When the numerical similarity between the 24th hour values of the 2nd group and the 30 th minute values of the 3 rd group attracted attention, these values were compared statistically with the one-way ANOVA method and it was concluded that there was no statistically significant difference between them $(p>0.05)$.

\section{Discussion}

In pelvic fractures, $85 \%$ of bleeding is caused by disruption of the integrity of the presacral venous plexus and bone $[13,14]$. Looking at the methods used to control bleeding in patients with pelvic fractures, we see non-invasive methods such as circumferential sheet wrapping, circumferential compression sling / binder device that allow temporary mechanical stabilization and reduce pelvic volume. These are the most common ones in the literature and can be easily applied by emergency teams at the time of diagnosis or suspicion of pelvic fracture [15-17].

Among the invasive methods of controlling bleeding, various pelvic external fixator applications are among the main methods. Bleeding control is aimed by temporarily providing pelvic stabilization with external fixator applications. It should be kept in mind that pelvic external fixator application is effective on bleeding control, but these applications should be performed by experienced and expert teams who know which technique to use in which fracture type [18]. Another invasive method, preperitoneal pelvic packing can be life-saving in cases with bleeding that cannot be controlled, however, it has been associated with deep tissue and wound infections as a complication afterwards [19]. 
Arterial injuries are seen at a rate of $10-15 \%$ in pelvis traumas. Transarterial embolization, recommended by the Eastern Association for the Surgery of Trauma guidelines, is an effective method that can be used in the control of arterial bleeding in patients with hemodynamically unstable pelvic fractures. Resuscitative Endovascular Balloon Occlusion of the Aorta (REBOA) technique is another arterial intervention that can be used in cases with uncontrolled bleeding originating from the arterial. It should be kept in mind that both methods have their own vascular and visceral complications [20,21].

The frequency of use of TRA in many surgical areas has increased, apart from orthopedic surgeries, because it reduces the amount of bleeding and the need for transfusion. Although it was first used in arthroplasty surgeries and elective spinal surgeries in orthopedic surgery, recently its use has come to the fore in femoral fractures, hip fractures, pelvis-acetabulum fractures and surgeries, which are known to be more bleeding from the fracture itself or due to the surgery to be performed. Although the literature gives information that the use of TRA in hip fracture surgeries will decrease the amount of bleeding and the need for transfusion, this situation has not been fully clarified in pelvis and acetabulum traumas. However, there is a consensus in these studies that the risk of thromboembolism does not change with the use of TRA [22].

In addition to elective surgeries in orthopedics, the frequency of use of TRA in fracture surgeries has increased in recent years. Studies revealing that the need for blood transfusion decreased with the use of TRA are in majority. Shaoyun Zhang et al. in their review of the literature to summarize the recent studies on TRA administration in traumatic orthopedic surgery, they concluded that topically applied
TRA in hip fracture surgery reduces blood loss without increasing the risk of thromboembolism and reduces the need for transfusion. In their review of the literature, they concluded that its effectiveness in other orthopedic surgeries such as pelvic and acetabular fractures is not clear [22]. In a metaanalysis of the use of TRA in patients with femur fracture, Pei Zhang et al. reported that both intravenous and topical TRA administration decreased the transfusion rate in femoral fracture surgery [23]. In their study to investigate the role of antifibrinolytics in pelvic and acetabular fracture surgery, Piggott RP et al. emphasized that in elective spinal and joint arthroplasty surgeries, antifibrinolytics provided benefits by reducing the amount of bleeding, but their role in trauma and fracture surgeries was not yet clear [24]. Spitler CA et al. conducted a randomized controlled trial to evaluate the safety and effectiveness of TRA use in pelvic ring, acetabulum and proximal femur fractures. They reported that the use of TRA in high-energy fractures of the pelvis, acetabulum, and femur significantly reduced the amount of bleeding, but did not decrease overall transfusion rates and also that the frequency of venous thromboembolism did not increase [25]. Watts $C D$ et al. reported in the randomized clinical trial they performed on patients who underwent hemi or total hip arthroplasty due to femoral neck fracture, the amount of blood loss and blood product transfusion rates in the TRA applied group were significantly decreased compared to the control group without TRA and that there was no difference between the two groups in terms of adverse effects within the first 30 days and 90 days after surgery [26].

Because unstable pelvic fractures can be fatal with massive bleeding, the intervention to be performed at the first encounter of patients with 
a pre-diagnosis of pelvic fracture in the area of trauma, such as pelvic binders and circumferential wrapping, which also allows temporary stabilization of the pelvis and can be easily applied by emergency teams, almost routinely started to be applied. In addition, prehospital application of TRA has come to the fore in order to reduce blood loss. Although there is no specific clinical study about the administration of TRA in patients with a prediagnosis of pelvic fracture in the literature, this issue has been included in the prehospital management of polytraumatized patients with acute bleeding, and studies suggesting early TRA administration in patients with uncontrolled bleeding have come to the fore. In this study, we created an acute bleeding trauma patient model by creating an unstable pelvic fracture in rats.

We aimed to see the effect of early TRA administration on hemoglobin value, without applying other measures that can be taken to reduce the amount of bleeding. When we look at the literature, in the Clinical Randomization of an Antifibrinolytic in Significant Hemorrhage 2, (CRASH-2) study, which is one of the most prominent studies on the subject, Shakur $\mathrm{H}$ et al. reported that administration of TRA within 1 hour significantly reduced the amount of bleeding and mortality [27]. Morrison JJ et al. retrospectively examined 896 patients who were injured during the war and had blood transfusion in Afghanistan, reported that although the mean injurity severity score was higher in 293 patients treated with TRA, the mortality was significantly lower [28]. In their retrospective study, El-Menyar A et al. compared 102 trauma patients who underwent prehospital TRA with the same number of trauma patients without TRA, they associated prehospital TRA with less need for blood transfusion. In their study, they demonstrated that there was no significant change in mortality rates and also thromboembolic events [29]. Levi $M$ evaluated the use of TRA in patients with severe trauma-related blood loss in a large international randomized controlled study, concluded that TRA administration reduced bleeding-related mortality, and that TRA did not cause thromboembolism and other serious adverse effects [30]. Neeki MM et al. identified injured patients who were exposed to penetrating or blunt trauma, with signs of hemorrhagic shock in prehospital evaluation, and who underwent prehospital TRA as the study group (362 patients in TRA group), and comprised the control group of injured patients who were exposed to penetrating or blunt trauma, with signs of hemorrhagic shock but did not receive TRA in the same center (362 patient in control group). When they compared the control group and the TRA group, they reported that mortality was $3.6 \%$ in the TRA group in the first 28 days of follow-up and $8.3 \%$ in the control group. When severely injured patients with ISS> 15 are compared, they reported that this difference is higher $(6 \%$ vs $14.5 \%$ for TRA and control, respectively). When they evaluated in terms of blood transfusion, they revealed that this need was significantly less in the TRA group. Similar to the literature, they also reported that there was no increase in side effects related to TRA [31]. In this experimental study we conducted, when the percent hemoglobin values at the beginning, 30 th minute and 24 th hour of group 3 were compared with each other, a statistically significant difference was found between these values, and this, first of all, showed us experimentally, that the decrease in hemoglobin values with the pelvis trauma continued significantly for 24 hours. This result is consistent with the information in the literature that there will be significant bleeding 
in unstable pelvic fractures. In our study, the 30 th minute values of group 2 (TRA after pelvic fracture) were found to be significantly higher than group 3 (no TRA after pelvic fracture). And also the percentage values of group 2 at the 24th hour after the trauma were found to be significantly higher than the percentage values of group 3. This result shows us that TRA application significantly reduces the amount of bleeding due to pelvic fracture. This result is consistent with the studies in the literature reporting that using TRA especially in the first hour after trauma reduces the amount of bleeding. In this context, our study supports studies stating that TRA can be used in the earliest period after trauma and even in the prehospital period, in addition to other interventions that can be applied to reduce bleeding, especially in traumas with acute bleeding such as pelvic fractures that are unstable and tend to bleed seriously. When we look back on the literature, we see that the side effects that may arise in the use of this easy-toapply, cost-effective agent in traumas with bleeding that may threaten the life of the patient are at a level that can be ignored depending on the gain to be achieved.

Another result that draws our attention in our study is that there is no statistically significant difference between the percentage values of the 24th hour of the second group in which TRA was applied after fracture, and the percentage values at the 30th minute of the 3rd group, who did not receive TRA after the fracture. The fact that the decrease observed in the 24-hour period in the TRA applied group occurred in only 30 minutes in the group without TRA, experimentally demonstrated a strong negative effect of TRA on bleeding. Whether it has the same strong effect in the clinic can only be determined by clinical studies.

\section{Conclusion}

In rats with bilateral unstable pelvic fractures due to blunt pelvis trauma, early administration of TRA after trauma significantly reduced the first 24-hour decrease in hemoglobin value. This experimental study supports the early and prehospital use of TRA in traumas that are predicted to progress with acute bleeding, such as unstable pelvic fractures. However, we think that more comprehensive clinical studies are needed to verify this and draw its boundaries.

Funding: The author(s) received no financial support for the research, authorship, and/or publication of this article.

Conflict of Interest: The authors declare that they have no conflict of interest.

\section{Ethical statement:}

The study was approved by Local Clinical Research Ethics Committee (Date and Decision Number: 2018/19), and written informed consent was obtained from each subject.

\section{Open Access Statement}

This is an open access journal which means that all content is freely available without charge to the user or his/her institution under the terms of the Creative Commons Attribution NonCommercial License (http://creativecommons.org/licenses/bync/4.0). Users are allowed to read, download, copy, distribute, print, search, or link to the full texts of the articles, without asking prior permission from the publisher or the author.

Copyright (c) 2021: Author (s).

\section{References}

[1]Haagsma JA, Graetz N, Bolliger I, et al. The global burden of injury: incidence, mortality, disability-adjusted life years and time trends from the Global Burden of Disease study 2013. Inj Prev. 2016;22(19:3-18. 
[2]Court-Brown CM, Caesar B. Epidemiology of adult fractures: a review. Injury. 2006;37(8):691-97.

[3]Holstein JH, Culemann U, Pohlemann T. What are predictors of mortality in patients with pelvic fractures? Clin Orthop Relat Res. 2012;470(8):2090-97.

[4]Giannoudis PV, Grotz MR, Tzioupis C, et al. Prevalence of pelvic fractures, associated injuries, and mortality: the United Kingdom perspective. J Trauma. 2007;63(4):875-83.

[5]Bakhshayesh P, Weidenhielm L, Enocson A. Factors affecting mortality and reoperations in high-energy pelvic fractures. Eur J Orthop Surg Traumatol. 2018;28(7):1273-82.

[6]Papakostidis C, Giannoudis PV. Pelvic ring injuries with haemodynamic instability: efficacy of pelvic packing, a systematic review. Injury. 2009;40: S53-61.

[7]Costantini TW, Coimbra R, Holcomb JB, et al. AAST Pelvic Fracture Study Group. Current management of hemorrhage from severe pelvic fractures: Results of an American Association for the Surgery of Trauma multi-institutional trial. J Trauma Acute Care Surg. 2016;80(5):717-23.

[8]Starr AJ, Griffin DR, Reinert CM, et al. Pelvic ring disruptions: prediction of associated injuries, transfusion requirement, pelvic arteriography, complications, and mortality. J Orthop Trauma. 2002; 16(8):553-61.

[9]Osborn PM, Smith WR, Moore EE, et al. Direct retroperitoneal pelvic packing versus pelvic angiography: A comparison of two management protocols for haemodynamically unstable pelvic fractures. Injury. 2009 Jan;40(1):54-60.

[10]Eastridge BJ, Starr A, Minei JP, et al. The importance of fracture pattern in guiding therapeutic decision-making in patients with hemorrhagic shock and pelvic ring disruptions. J Trauma. 2002;53(3):446-50.

[11]Chauncey JM, Wieters JS. Tranexamic Acid. 2021 Mar 2. In: StatPearls [Internet]. Treasure Island (FL): StatPearls Publishing; 2021 Jan.

[12] Vermylen J, Verhaegen-Declercq L, Verstraete M, et al. A double blind study of the effect of tranexamic acid in essential menorrhagia. Thromb Haemost. 1968;20(3):583-87.

[13]Balogh Z, Caldwell E, Heetveld M, et al. Institutional practice guidelines on management of pelvic fracture-related hemodynamic instability: do they make a difference? J Trauma. 2005;58(4):778-82.

[14]Jain S, Bleibleh S, Marciniak J, et al. A national survey of United Kingdom trauma units on the use of pelvic binders. Int Orthop. 2013;37(7):1335-39.

[15] Ooi CK, Goh HK, Tay SY, et al. Patients with pelvic fracture: what factors are associated with mortality? Int J Emerg Med. 2010;3(4):299-304.

[16] Koo H, Leveridge M, Thompson C, et al. Interobserver reliability of the youngburgess and tile classification systems for fractures of the pelvic ring. J Orthop Trauma. 2008;22(6):379-84.

[17] Manson T, O'Toole RV, Whitney A, et al. Young-Burgess classification of pelvic ring fractures: does it predict mortality, transfusion requirements, and nonorthopaedic injuries? J Orthop Trauma. 2010;24(10):603-39.

[18] Stahel PF, Mauffrey C, Smith WR, et al. External fixation for acute pelvic ring injuries: decision making and technical options. J Trauma Acute Care Surg. 2013;75(5):882-87. 
[19]Filiberto DM, Fox AD. Preperitoneal pelvic packing: Technique and outcomes. Int $\mathrm{J}$ Surg. 2016;33(Pt B):222-24.

[20] Awwad A, Dhillon PS, Ramjas G, et al. Trans-arterial embolisation (TAE) in haemorrhagic pelvic injury: review of management and mid-term outcome of a major trauma centre. CVIR Endovasc. 2018;1(1):32.

[21] Osborn LA, Brenner ML, Prater SJ, et al. Resuscitative endovascular balloon occlusion of the aorta: current evidence. Open Access Emerg Med. 2019;11:29-38.

[22]Zhang S, Xiao C, Pei F. Research progress on tranexamic acid in traumatic orthopedic surgery. Zhongguo Xiu Fu Chong Jian Wai Ke Za Zhi. 2019;33(11):1457-61.

[23]Zhang P, Bai J, He J, et al. A systematic review of tranexamic acid usage in patients undergoing femoral fracture surgery. Clin Interv Aging. 2018;13:1579-91.

[24]Piggott RP, Leonard M. Is there a role for antifibrinolytics in pelvic and acetabular fracture surgery? Ir J Med Sci. 2016;185(1):29-34.

[25] Spitler CA, Row ER, Gardner WE 2nd, et al. Tranexamic Acid Use in Open Reduction and Internal Fixation of Fractures of the Pelvis, Acetabulum, and Proximal Femur: A Randomized Controlled Trial. J Orthop Trauma. 2019;33(8):371-76.

[26] Watts CD, Houdek MT, Sems SA, et al. Tranexamic Acid Safely Reduced Blood Loss in Hemi- and Total Hip Arthroplasty for Acute Femoral Neck Fracture: A Randomized Clinical Trial. J Orthop Trauma. 2017;31(7):345-51.

[27]CRASH-2 trial collaborators, Shakur H, Roberts I, Bautista R, et al. Effects of tranexamic acid on death, vascular occlusive events, and blood transfusion in trauma patients with significant haemorrhage
(CRASH-2): a randomised, placebocontrolled trial. Lancet. 2010;376(9734):2332.

[28] Morrison JJ, Dubose JJ, Rasmussen TE, et al. Military Application of Tranexamic Acid in Trauma Emergency Resuscitation (MATTERs) Study. Arch Surg. 2012;147(2):113-19.

[29]El-Menyar A, Sathian B, Wahlen BM, et al. Prehospital administration of tranexamic acid in trauma patients: A 1:1 matched comparative study from a level 1 trauma center. Am J Emerg Med. 2020;38(2):26671.

[30]Levi M. Should antifibrinolytics be given in all patients with trauma? Curr Opin Anaesthesiol. 2012;25(3):385-88.

[31] Neeki MM, Dong F, Toy J, et al. Tranexamic Acid in Civilian Trauma Care in the California Prehospital Antifibrinolytic Therapy Study. West J Emerg Med. 2018;19(6):977-86. 